\title{
Philosophiques
}

\section{Les qualités selon Stout}

\section{Claude Panaccio}

Volume 13, numéro 2, automne 1986

URI : https://id.erudit.org/iderudit/203318ar

DOI : https://doi.org/10.7202/203318ar

Aller au sommaire du numéro

\section{Éditeur(s)}

Société de philosophie du Québec

ISSN

0316-2923 (imprimé)

1492-1391 (numérique)

Découvrir la revue

Citer cet article

Panaccio, C. (1986). Les qualités selon Stout. Philosophiques, 13(2), 237-252.

https://doi.org/10.7202/203318ar

\section{Résumé de l'article}

George Frederick Stout fut, dans la philosophie du XX $\mathrm{X}^{\mathrm{e}}$ siècle, le principal promoteur de la thèse à tendance nominaliste du particularisme des qualités, selon laquelle chaque propriété d'une entité individuelle quelconque est elle-même une entité individuelle. On examine ici de façon critique les arguments avancés par Stout en faveur de cette doctrine ontologique ainsi que les objections qui lui ont été adressées. N'ayant trouvé au bout du compte aucun argument décisif ni d'un côté ni de l'autre, on suggère, dans une veine inspirée de Carnap, de réinterpréter la thèse originale comme une recommandation relative à l'adoption d'un certain langage.
Ce document est protégé par la loi sur le droit d'auteur. L’utilisation des services d'Érudit (y compris la reproduction) est assujettie à sa politique d'utilisation que vous pouvez consulter en ligne.

https://apropos.erudit.org/fr/usagers/politique-dutilisation/ 


\title{
LES QUALITÉS SELON STOUT *
}

\author{
par Claude Panaccio
}

\begin{abstract}
RÉSUMÉ. George Frederick Stout fut, dans la philosophie du $\mathrm{XX}^{\mathrm{e}}$ siècle, le principal promoteur de la thèse à tendance nominaliste du particularisme des qualités, selon laquelle chaque propriété d'une entité individuelle quelconque est elle-même une entité individuelle. On examine ici de façon critique les arguments avancés par Stout en faveur de cette doctrine ontologique ainsi que les objections qui lui ont été adressées. N'ayant trouvé au bout du compte aucun argument décisif ni d'un côté ni de l'autre, on suggère, dans une veine inspirée de Carnap, de réinterpréter la thèse originale comme une recommandation relative à l'adoption d'un certain langage.
\end{abstract}

ABSTRACT. George Frederick Stout was, in twentieth century philosophy, the leading defender of the nominalistically oriented thesis of the particularisation of qualities, according to which each property of an individual is itself an individual. This paper is a critical discussion of the arguments put forward by Stout for this ontological view and of the objections raised by his opponents. Since no decisive argument is found on either side, it is suggested, in a Carnapian mood, to reconstruct the original thesis as a proposal for the adoption of a certain language.

À propos de son article «On denoting», dont on a dit parfois qu'il a révolutionné la philosophie contemporaine, Bertrand Russell écrit dans son Histoire de mes idées philosophiques:

La théorie des descriptions (...) fut exposée pour la première fois dans mon article «sur la désignation» que Mind publia en 1905. Cette théorie parut au rédacteur en chef d'alors si absurde qu'il me supplia

* Une première version de ce texte a été présentée au XIe congrès de la Société de Philosophie du Québec, le 11 mai 1984 à Québec. La recherche dont il est le fruit a été appuyée par les subventions 410-83-0306 et 410-84-0569 du Conseil de recherches en sciences humaines du Canada. Je remercie tout spécialement mon assistant de recherche, M. Yves Bastarache, d'avoir procédé à une exploration préliminaire de certaines parties du corpus. 
de reconsidérer ma décision de le publier et de ne pas exiger sa publication tel qu'il était. Pour ma part, cependant, j'étais persuadé de sa justesse et je refusai de céder. (Russell, 1961, 103-4).

C'est à un philosophe britannique du nom de George Frederick Stout (1860-1944) que renvoie cette description définie: «le rédacteur en chef de la revue Mind en 1905». Il n'est pas présenté sous un jour très favorable dans ce passage de Russell, mais on peut aussi se dire avec Robert Marsh qu'après tout, malgré des réticences bien compréhensibles pour l'époque, Stout eut en fin de compte le bon jugement de publier «On denoting» dans sa version intégrale 1 .

Ce n'est pas son seul titre de gloire. Russell, dans son autobiographie, attribue à Stout et à McTaggart la responsabilité de l'avoir " plongé» dans l'idéalisme alors qu'il était étudiant ${ }^{2}$. Et l'importante Encyclopedia of Pbilosophy de Paul Edwards lui consacre un article de quatre colonnes:

Il n'y a probablement, écrit l'auteur, aucun philosophe qui, dans sa façon de penser, ait accompli de manière aussi coulante la transition entre l'idéalisme qui dominait à la fin du dix-neuvième siècle et la philosophie critique et non spéculative qui dominait au milieu du vingtième. (Mace, 1967, 24; ma traduction).

La thèse de Stout que je discuterai ici n'a en tout cas rien de spécialement idéaliste. Elle a été exposée par lui dans un certain nombre d'articles postérieurs à la période qu'évoque Russell, les trois principaux d'entre eux datant respectivement de 1921, 1923 (à l'occasion d'une table-ronde où il eut à répondre aux critiques du redoutable G.E. Moore) et 1936. Dans sa version radicale, cette thèse, que j'appelle le particularisme des qualités, veut que les qualités des individus particuliers soient elles-mêmes des entités individuelles particulières. Déjà au quatorzième siècle, Guillaume d'Occam avait fait de cette doctrine l'une des pièces maîtresses de son nominalisme ${ }^{3}$ et maints commentateurs en retracent l'origine

1. In Russell (1956), p. 39

2. RuSSELL (1968), p. 170.

3. L'édition critique des œuvres philosophiques et théologiques de Guillaume d'Occam est maintenant complétée (Occam, 1967-86). Certaines bonnes traductions anglaises sont disponibles (Occam, 1957, 1969, 1973-74, 1974, 1980). J'ai, de mon côté, consacré plusieurs travaux à la sémantique et à l'ontologie du «venerabilis inceptor» (Panaccio, 1972, 1973, 1974, 1980, 1981, 1983, 1984, 1985) et c'est justement ce qui 
jusqu'à Aristote lui-même ${ }^{4}$. Aujourd'hui, le nom de Stout reste encore étroitement associé, dans les débats sur le nominalisme, au particularisme des qualités ${ }^{5}$ et bien que, sous une forme ou sous une autre, l'idée ait été défendue aussi par des philosophes plus récents ${ }^{6}$, je me limiterai dans le présent article à l'examen critique de la position de Stout.

\section{La thèse}

En voici la formulation de 1921:

Un caractère (character) caractérisant une chose concrète ou un individu est aussi particulier que la chose ou l'individu qu'il caractérise. De deux boules de billard, chacune a sa propre rondeur séparée et distincte de celle de l'autre, tout comme les boules de billard ellesmêmes sont distinctes et séparées. (Stout, 1966, 7; ma traduction).

Cela ne signifie pas qu'il doive nécessairement y avoir quelque subtile différence qualitative entre ces deux rondeurs. Stout maintient au contraire qu'elles peuvent en principe se ressembler avec une parfaite exactitude ${ }^{7}$. Il ne parle que de différence numérique. Quelle que soit leur ressemblance, affirme-t-il, la rondeur de cette boule de billard est une entité individuelle distincte de la rondeur de cette autre boule de billard, pour peu justement que les boules en question soient elles-mêmes distinctes l'une de l'autre. Et il en va de même, selon lui, de toutes les propriétés des objets individuels: chacun a les siennes propres. Chaque propriété est une entité individuelle qui ne peut être

m'a poussé à examiner de plus près la discussion contemporaine sur le particularisme des qualités.

4. Sur le particularisme des qualités chez Aristote, voir ACKrill (1963), AlLEN (1969), Anscombe et Geach (1967), Matthews et Cohen (1967-68). Cette interprétation d'Aristote est cependant contestée (à tort, me semble-t-il) par Owen (1965).

5. Par exemple : «... G.F. Stout (...), with whom the doctrine is most closely associated in twentieth-century Anglo-saxon philosophy..." (ARMSTRONG, 1978, p. 77; la doctrine dont il est question ici est bien le particularisme des qualités). Ou encore: "The best known and most extreme defender of particularised attributes, G.F. Stout...» (LeVINSON, 1980, p. 102). «In modern times, it was G.F. Stout who first explicitly made the proposal that properties and relations are as particular as the substances that they qualify». (CAMPBELL, 1981, p. 477).

6. Diverses formes de particularisme des qualités apparaissent chez CAMPBELL (1981), Cocchiarella (1972), Goodman (1977), Levinson (1980), Williams (1953), WOLTERSTORFF (1970).

7. Stout (1923), pp. 119-20. 
associée qu'à un objet et un seul, mais qui est néanmoins distincte de cet objet. Et il en va de même encore des relations entre entités individuelles: une relation entre $a$ et $b$ ne peut jamais être identique à une relation entre $c$ et $d$, bien que l'une et l'autre puissent en principe se ressembler parfaitement.

Les qualités et les relations, cependant, tout comme les substances, se laissent regrouper en classes ou en sortes (classes or kinds). La couleur de cette balle de ping-pong appartient à la même sorte de couleurs que la couleur de ce banc de neige : ce sont deux blancheurs. Et la forme de cette balle de ping-pong appartient à la même sorte de formes que la forme de cette boule de billard: ce sont deux rondeurs. Tout se passe ici comme pour deux chiens : ils appartiennent tous deux à la même sorte d'animaux. Aux yeux de Stout, ces classes naturelles ou sortes d'entités, qu'il s'agisse de sortes de qualités, de sortes de relations ou de sortes de substances, sont des unités ontologiques primitives, irréductibles aux entités individuelles qui en font partie. Il les appelle des "unités distributives » ${ }^{8}$. C'est justement par leur admission au titre d'entités irréductibles que Stout se sépare du nominalisme radical d'un Guillaume d'Occam. Je n'explorerai pas davantage ici cet aspect réaliste de la pensée de Stout pour concentrer plutôt la discussion sur la thèse à saveur nettement nominaliste du particularisme des qualités.

\section{Les arguments de Stout}

Au fil de ses écrits sur la question, je dénombre essentiellement quatre arguments invoqués par Stout. Je les examinerai tour à tour.

2.1. Le premier, l'argument par les états transitoires (transient states), n'a qu'une visée restreinte. Il veut seulement montrer que certaines propriétés au moins sont des entités particulières, pour ébranler au départ ceux qui d'emblée seraient convaincus de ce que toutes les propriétés sont des universaux. La première prémisse constate que les états transitoires, comme le vol d'un oiseau, l'explosion d'une mine ou encore - et c'est là l'exemple fétiche dans cette discussion - un reniflement, sont de

8. Stout (1966), p. 7; (1936), p. 3. 
toute évidence des particuliers et non des universaux. La deuxième prémisse affirme que les états transitoires sont des propriétés ou des relations. Par conséquent, conclut Stout, certaines propriétés ou relations sont des particuliers et non des universaux?

Cet argument est formellement correct. Et la majeure est, en un sens, indubitable: un reniflement donné est quelque chose de numériquement distinct de tout autre reniflement. Mais la mineure, elle, est bien vulnérable et c'est ce que les critiques ont à juste titre exploité depuis la contribution, publiée en 1923, de George Edward Moore à un symposium avec Stout sur le particularisme des qualités, que Moore pour sa part rejette entièrement. Un reniflement, faisait-il remarquer, n'est pas une propriété, mais un événement ${ }^{10}$. Récemment encore, Armstrong (1978) répond de la même manière au même argument de Stout en invoquant, pour sa part, la notion d'état de choses (state of affairs) ${ }^{11}$. Un état de choses, c'est le fait qu'un individu donné ait à tel moment une certaine propriété ou que tels ou tels individus donnés entretiennent à tel moment une certaine relation. Cela est bel et bien un particulier et non un universel, mais il n'en suit guère que la propriété ou la relation qui entre en jeu dans l'état de choses ou dans l'événement soit elle-même un particulier. Certes le reniflement de Monsieur Untel à tel moment est un état de choses particulier; il n'en découle pas, observent les réalistes, que la propriété de renifler soit elle-même autre chose qu'un universel. Cette critique est dirimante et il faut certainement renoncer à invoquer en faveur du particularisme des qualités la particularité des reniflements. Les réalistes n'en sont nullement ébranlés dès lors qu'ils disposent d'une notion d'《événement»» ou d'《état de choses» distincte de celle de «propriété».

2.2. Stout invoque, en deuxième lieu, l'argument par la localisation spatiale. Il faut bien admettre, soutient-il, que la rondeur de cette boule de billard est localisée en un endroit différent de la rondeur de cette autre boule de billard. Chacune circonscrit sa propre portion d'espace. Or comment une même entité, la rondeur, pourrait-elle tout entière être ainsi localisée

\footnotetext{
9. STOUT (1966), pp. 9-10.

10. MOORE (1962), pp. 19-20.

11. ARMSTRONG (1978), p. 80.
} 
dans deux endroits distincts? Cela est impossible. Et donc chaque rondeur d'un objet particulier est elle-même une entité particulière ${ }^{12}$.

La réponse classique des adversaires de Stout est, ici aussi, péremptoire ${ }^{13}$. C'est une pure pétition de principe que d'appliquer aux propriétés et aux relations le principe de non-ubiquité, qui ne saurait concerner dans sa formulation même que les entités individuelles. Un universel peut très bien, pour le réaliste, être simultanément associé à plusieurs lieux distincts en ce sens précis qu'il peut être simultanément exemplifié par des entités individuelles sises en des lieux divers. Ce deuxième argument de Stout fait donc long feu, lui aussi.

2.3. Le troisième peut être considéré comme son argument principal, «my general argument», dit-il ${ }^{14}$. Il n'est malheureusement jamais formulé de façon très nette et les critiques hésitent quant à la façon de l'interpréter. Le point de départ en est qu'une substance n'est rien sans ses propriétés. Stout prend acte de la critique classique de l'idée d'une substance pure qui existerait en elle-même sous les propriétés. Il en conclut qu'une substance particulière n'est en réalité qu'un agrégat de propriétés ( $a$ bundle of properties»). Or, poursuit l'argument, si les propriétés étaient des universaux, on ne voit aucunement comment elles pourraient en se combinant finir par engendrer du particulier. Cela demeurerait tout à fait inintelligible. Si les propriétés sont des universaux, chacune doit en principe pouvoir être exemplifiée par une pluralité d'entités individuelles; c'est là la définition même d'un universel. Et cela doit donc demeurer vrai de n'importe quel amalgame de telles propriétés universelles. On n'arrivera jamais par cette voie à rejoindre la singularité. Bref, si un objet concret singulier n'est jamais rien d'autre qu'un agrégat de propriétés, il faut qu'au départ ces propriétés soient elles-mêmes des entités singulières, ce qui est justement la thèse du particularisme des qualités.

12. Stout (1966), pp. 10-11; (1936), pp. 10-11.

13. MOORE (1962), p. 25; ARMSTRONG (1978), p. 79 ; LEVINSON (1980), p. 103.

14. Stout (1966), pp. 11-12. 
Cet argument doit faire face à au moins deux sortes d'objections. On peut d'abord faire remarquer qu'il repose, sans argumentation spéciale, sur une négation du principe, souvent admis en philosophie, de l'identité des indiscernables. Selon ce principe, si toute propriété de $\mathrm{x}$ est une propriété de $\mathrm{y}$, alors $\mathrm{x}$ est identique à y. Or l'argument admet de son côté que n'importe quelle combinaison de propriétés générales puisse en principe être exemplifiée plus d'une fois. Je ne m'engagerai pas plus avant dans ce périlleux débat, mais il faudrait certainement se demander si les propriétés dont parle le principe de l'identité des indiscernables se limitent bien aux universaux, c'est-à-dire aux propriétés exemplifiables en principe par plusieurs individus distincts. $A$ défaut de quoi, bien sûr, le principe ne serait plus inconciliable avec l'argumentation de Stout.

La seconde façon de contrer l'argument est, cependant, plus efficace. Elle consiste à rejeter la conception de la chose concrète comme agrégat de propriétés. Cette idée a sans doute connu son heure de gloire au moment où Stout écrivait, mais elle est beaucoup moins facilement admise aujourd'hui. Pour Kripke par exemple, l'identité d'une chose concrète singulière apparaît comme une donnée primitive qu'on n'éclaire aucunement par l'idée d' «agrégat» de propriétés ${ }^{15}$. Le retour récent d'un certain aris totélisme dans la philosophie analytique désamorce l'argument de Stout. On peut même, comme l'indiquait déjà Moore, admettre la critique traditionnelle de l'idée de substrat pur sans se commettre pour autant à la doctrine de la singularité comme agrégat de propriétés. Tout ce qu'on doit admettre, rappelle Moore, c'est qu'une chose singulière $a$ toujours des propriétés; il n'en suit nullement qu'elle soit ses propriétés ${ }^{16}$. L'argument principal échoue donc, lui aussi.

2.4. Le quatrième argument est purement négatif. Stout trouve carrément inintelligible l'idée qu'il existe des propriétés universelles à titre d'entités distinctes, "a frightfully difficult view to understand ${ }^{17}$. Il faudrait dire par exemple, ironise-t-il,

15. Voir KRIPKE $(1971,1980)$. Sur le déclin de l'idée d'agrégat de propriétés dans la philosophie analytique récente, voir Loux (1983), pp. 14-16.

16. MOORE (1962), pp. 29-30.

17. Stout (1923), p. 118. 
que la forme de la boule de billard est une entité réelle distincte de sa rondeur, puisqu'il y aurait là deux universaux distincts: la forme en général et la rondeur en général. Stout, lui, s'avoue incapable de distinguer la forme d'une boule de billard de la rondeur de cette même boule: "I can do notbing of the sort." 18 Toute la question serait donc de savoir si le réalisme des universaux est une thèse défendable. Qu'il suffise ici de remarquer que, de toute façon, on ne trouve guère là un argument positif en faveur du particularisme des qualités. On pourrait rejeter l'idée que les propriétés sont des entités universelles distinctes sans devoir admettre pour autant qu'elles sont des entités particulières distinctes. Pourquoi devraient-elles absolument être des entités distinctes?

Bref, on ne peut retenir, au bout de la ligne, aucun argument qui appuie de façon spécifique et suffisante la doctrine du particularisme des qualités. Cela ne signifie pas qu'elle soit fausse, mais nous n'avons encore trouvé aucune bonne raison de l'adopter.

\section{Les arguments contre Stout}

En avons-nous pour la rejeter? Je considérerai brièvement trois arguments intéressants qui ont été avancés pour réfuter le particularisme des qualités. Les deux premiers se trouvent déjà chez Moore. J'emprunte le dernier à Armstrong (1978).

3.1. Dans sa version la plus dénudée, la première objection se contente tout simplement de recourir à l'évidence: «quite certainly, dit Moore, many characters of concrete things are not particular» ${ }^{19}$. Deux choses concrètes distinctes ont très souvent des propriétés communes, comme d'être toutes les deux rondes. Il est donc parfaitement évident qu'il existe des propriétés communes.

Non sans raison, Stout voit là une pétition de principe :

M. Moore n'est pas d'accord, écrit-il. Et puisque c'est M. Moore qui n'est pas d'accord, j'attache de l'importance à son désaccord. Mais il n'a en réalité rien fait d'autre que de signaler qu'il n'était pas d'accord. (Stout, 1923, 120; ma traduction).

18. lbid., p. 118.

19. MOORE (1962), p. 30. 
L'argument de Moore, cependant, peut être réinterprété comme un appel au langage ordinaire. Nous disons tout naturellement que deux boules de billard ont la même forme, que cette voiture est de la même couleur que cette autre, et ainsi de suite. Et donc nous reconnaissons déjà, comme locuteurs du langage ordinaire, l'existence des propriétés communes. Ainsi considéré, c'est un argument qui porte; mais il ne fournit qu'une simple présomption. Le langage ordinaire, il est vrai, admet bel et bien les propriétés communes, mais il n'est pas lui-même au-dessus de tout soupçon. Le résultat de l'argument serait ainsi de renvoyer le fardeau de la preuve du côté du particularisme des qualités, ce qui en pareille matière est loin d'être négligeable. Néanmoins, il ne le réfuterait pas de manière décisive.

La réponse de Stout est double. D'une part, il essaie de montrer que le langage ordinaire ne lui est pas si hostile ${ }^{20}$. Après tout, nous disons bien que la couleur de cette pièce de tissu est exactement semblable à celle de cette autre, ce qui suggère fortement, selon Stout, que les deux couleurs sont des entités numériquement distinctes; sans quoi, il serait inapproprié de parler de similitude entre elles. Cette réponse est incorrecte. $\mathrm{La}$ similitude n'est pas en général considérée comme une relation irréflexive et il ne suit nullement de « $\mathbf{x}$ ressemble à $\mathbf{y}$ » que $\mathbf{x}$ soit distinct de y. En tout cas, le locuteur ordinaire qui affirme que la couleur de cette pièce est exactement semblable à celle de cette autre n'admettrait sans doute pas facilement qu'il y ait donc là deux couleurs distinctes! Au contraire, insisterait-il, je veux justement dire que les deux pièces sont exactement de la même couleur. La présomption du langage ordinaire demeure bel et bien du côté des propriétés communes.

L'autre réponse de Stout est que ces façons ordinaires de parler qu'on invoque contre lui peuvent être vues comme des abréviations commodes qu'il ne faut pas trop prendre à la lettre ${ }^{21}$. Le phénomène est courant, observe-t-il, lorsqu'il s'agit d'identité. Ainsi nous disons facilement qu'un même événement se répète plusieurs fois ou que les mêmes causes engendrent les mêmes effets alors que nous savons très bien qu'à strictement

20. STOUT (1936), p. 10.

21. Ibid., p. 4. 
parler chaque événement, chaque cause, chaque eifet est unique. Nous allégeons seulement notre discours en utilisant "même» un peu à toutes les sauces, là où un langage tout à fait précis requerrait une pléiade de relations d'équivalence plus complexes, comme la similitude, l'appartenance à une même classe, etc. C'est ainsi que nous parlons du même autobus pour des véhicules distincts mais fonctionnellement équivalents; ou de la même heure - par exemple dans l'expression «demain, à la même heure» - pour deux moments du temps que nous savons très bien différencier l'un de l'autre. Pourquoi n'en irait-il pas ainsi des expressions comme «la même couleur» ou «la même forme»?

Cette réponse ne montre qu'une chose, c'est qu'on peut, lorsqu'on a de bonnes raisons de le faire, renoncer aux identités suggérées par les usages ordinaires du mot « même». Mais elle ne fournit, notons-le, aucune raison spécifique de renoncer à l'identité des propriétés communes. La réponse de Stout peut bien ébranler celui qui croit devoir toujours prendre le langage ordinaire au pied de la lettre, mais elle ne suffit pas à conjurer tout à fait la présomption originale. Si l'argument du langage ordinaire ne réfute pas le particularisme des qualités, son résultat net, cependant, est de lui renvoyer le fardeau de la preuve, ce qui n'est pas rien.

3.2. Voici une deuxième objection également due à Moore. Si la rondeur de l'objet $A$ était réellement distincte de la rondeur de l'objet $B$, on ne comprendrait pas qu'on puisse dire de manière univoque que $A$ est rond et que $B$ est rond. Il faudrait que le sens du mot «rond» varie d'un cas à l'autre, puisqu'il renverrait chaque fois à une rondeur différente 22 .

Je trouve la réponse de Stout parfaitement convaincante ${ }^{23}$. Le particularisme des qualités n'entraîne aucunement l'équivocité d'une expression prédicative comme «est rond». Dans son interprétation par exemple, dire que $A$ est rond, c'est dire que $A$ appartient à la classe des objets qui ont une rondeur particulière, et dire que $\mathrm{B}$ est rond, c'est dire exactement la même chose à

22. MOORE (1962), pp. 20-21.

23. Stout (1923), pp. 118-119. 
propos de B. Il n'y a là aucune équivocité. L'argument de Moore échoue ici complètement, victime d'une conception Fido-Fido du langage, selon laquelle à chaque expression linguistique doit correspondre dans la réalité une entité et une seule.

3.3 Armstrong (1978) avance contre Stout deux objections de son cru, mais comme il admet lui-même que la première n'est pas très décisive ${ }^{24}$ et que je ne lui vois, pour ma part, guère de force, je me contenterai de discuter ici la seconde, qui nous est, celle-là, présentée comme déterminante ${ }^{25}$. Le particularisme des qualités, prétend-elle, n'arrive pas à éliminer les propriétés universelles, il ne réussit au mieux qu'à les reculer au niveau des propriétés de propriétés. Supposons en effet que la blancheur d'un objet singulier soit elle-même une entité singulière. Cette entité aurait à son tour certaines propriétés, elle serait par exemple une couleur, elle aurait une certaine teinte, et ainsi de suite. Si ces propriétés de la blancheur singulière de tel objet singulier étaient elles-mêmes des entités singulières distinctes, elles devraient notamment être distinctes de la blancheur en question, laquelle ne serait donc en elle-même que pure particularité (bare particular). Considérées strictement en elles-mêmes, les propriétés de premier ordre seraient de la sorte indiscernables les unes des autres, puisque toute propriété par laquelle l'une d'elles pourrait se distinguer des autres ne serait jamais ellemême qu'une autre singularité, extrinsèque donc par rapport à la propriété originale. Il faut donc, poursuit l'argument, que les propriétés de propriétés soient des universaux et non des particuliers. Et il n'en faut pas plus! Le réaliste trouve là tous les universaux dont il puisse rêver. La propriété d'être le jaune de ce citron, par exemple, serait un universel, puisque c'est une propriété de propriété. «À ce point, conclut Armstrong, je pense que la conception particulariste des propriétés et des relations doit être définitivement et complètement abandonnée » ${ }^{26}$.

L'objection, cependant, n'est pas si dévastatrice. Stout avait prévu le coup. Il soutient explicitement qu'à la différence des choses concrètes, les propriétés et les relations n'appartiennent

24. Armstrong (1978), p. 86.

25. Ibid., pp. 86-87.

26. Ibid., p. 87 (ma traduction). 
pas à des classes ou à des sortes parce qu'elles ont elles-mêmes des propriétés, mais plutôt parce qu'elles sont des propriétés ou des relations ${ }^{27}$. Les propriétés singulières de premier ordre sont, à ses yeux, des atomes ontologiques: chacune st distingue de chaque autre par elle-même et non par le biais de ses propres propriétés. La contre-attaque est efficace. Toute ontologie doit finir par admettre des entités qui sont distinctes des autres par ce qu'elles sont plutôt que par le biais d'autres choses. Qu'Armstrong veuille à tout prix que les propriétés de premier ordre ne se différencient ontologiquement les unes des autres que par des propriétés de second ordre montre simplement qu'il refuse d'emblée aux propriétés de premier ordre le statut d'entités primitives. Son argument repose donc sur une pétition de principe.

\section{4. Épilogue carnapien}

Le bilan de la discussion paraît assez mince. Nous n'avons pas trouvé chez Stout de bon argument qui soit spécifiquement en faveur du particularisme des qualités et nous n'avons pas trouvé chez ses adversaires d'objection décisive pour le réfuter. Tout ce qui reste, c'est une raison prima facie de ne pas adopter le particularisme des qualités: le langage ordinaire nous pousse dans l'autre sens et rien, apparemment, ne vient faire contrepoids. Le fardeau de la preuve est donc du côté du particularisme des qualités et le défi n'est pas adéquatement relevé par Stout.

Faut-il en rester là ? Peut-être pouvons-nous jeter un éclairage nouveau sur l'ensemble de la question en la considérant d'un cil carnapien. Carnap (1937) soutient que très souvent les thèses philosophiques du genre de celles que nous scrutons ici gagneraient à être interprétées comme des recommandations, des "proposals", pour l'adoption de certains langages ou de certaines façons de parler:

Tout débat, écrit-il, sur la vérité ou la fausseté d'une telle thèse est complètement inapproprié, une pure bataille de mots ; nous pouvons au mieux discuter l'utilité de la suggestion ou en examiner les conséquences. (Carnap, 1937, 299-300; ma traduction).

27. Stout (1923), p. 116. 
Et Stout ne se dit-il pas lui-même porté à croire que la différence entre Moore et lui pourrait bien être au fond «une différence de langage ${ }^{28}$ ? Reconstruit dans cette veine, le particularisme des qualités deviendrait une recommandation pour l'adoption $a ̀$ des fins théoriques ${ }^{29}$ d'un langage dans lequel les termes abstraits de propriétés ou de relations comme «blancheur», "rondeur» ou "paternité» seraient des termes généraux plutôt que des noms propres. On y utiliserait plus volontiers le pluriel: "les blancheurs », «les rondeurs», «les paternités». On dirait « les rondeurs sont des formes» au lieu de "la rondeur est une forme»; ou encore «ces deux balles ont des formes de la même sorte» plutôt que «ces deux balles ont la même forme».

Cette suggestion semble praticable. Les critiques adressées à Stout ne montrent qu'une chose, c'est que le langage qu'il propose n'est pas celui que nous parlons ordinairement. Mais elles n'y décèlent aucune véritable incohérence logique. La question, dès lors, serait de savoir ce qu'on gagnerait à l'adoption d'un tel idiome stoutien. Notre discussion suggère qu'aucun avantage n'a été mis en lumière par les arguments de Stout lui-même. Si on accepte la transposition carnapienne, la façon de poursuivre le débat serait sans doute de construire un langage stoutien en modèle réduit et d'en comparer les capacités expressives avec celles de certains modèles réduits correspondants de langages réalistes d'une part (où une expression comme «la blancheur» fonctionne comme un nom propre) et de langages nominalistes radicaux d'autre part (où ni les noms de classe ou de sorte, ni les noms de propriété ne sont des noms propres). Une telle confrontation requerrait, à n'en pas douter, un appareil d'analyse sémantique beaucoup plus sophistiqué que celui dont disposait Stout.

Département de philosophie,

Université du Québec à Trois-Rivières.

28. Stout (1936), p. 15.

29. Cf. Stout (1936), p. 4: «... even when we have made the analysis, it would be intolerably cumbersome to substitute this for the less exact language of daily life». 


\section{REFERENCES BIBLIOGRAPHIQUES}

ACKRILL, J.L. (1963), Aristotle's Categories and De Interpretatione (traductions anglaises annotées), Oxford, Clarendon Press.

ALLEN, R.E. (1969), "Individual properties in Aristotle's Categories", Phronesis, 14, pp. 31-39.

ANSCOMBE, G.E.M. et GEACH, P.T. (1967), Three Philosophers, Oxford, Basil Blackwell.

ARMSTRONG, D.M. (1978), Nominalism and Realism (=Universals and Scientific Realism, vol. I), Cambridge, Cambridge University Press.

CAMPBELL, K. (1981), « The metaphysics of abstract particulars », in Midwest Studies in Philosophy VI: The Foundations of Analytic Philosophy, sous la dir. de P.A. French et als., Minneapolis, University of Minnesota Press, Pp. 477-488.

CARNAP, Rudolf (1937), The Logical Syntax of Language (trad. angl. par A. Smeaton), Londres, Routledge and Kegan Paul ( $1^{\text {re }}$ éd. allemande: 1934).

COCCHIARELLA, N.B. (1972), « Properties as individuals in formal ontology ", Noûs, 6, pp. 165-187.

GOODMAN, N. (1977), The Structure of Appearance, Dordrecht, Reidel ( $1^{\text {re }}$ éd. : Cambridge, Mass., Harvard University Press, 1951).

KNIGHT, H. (1936), «Stout on universals», Mind, 177, pp. 45-60.

KRIPKE, S. (1971), "Identity and necessity», in Identity and Individuation, sous la dir. de M.K. Munitz, New York, New York University, pp. 135-164 (repris in Naming, Necessity, and Natural Kinds, sous la dir. de S.P. Schwartz, Ithaca, Cornell University Press, 1977, pp. 66-101).

KRIPKE, S. (1980), Naming and Necessity, Cambridge, Mass., Harvard University Press, (1 ${ }^{\text {re }}$ éd. in Semantics of Natural Language, sous la dir. de D. Davidson et G. Harman, Dordrecht, Reidel, 1972, pp. 253-355; tr. franç. par P. Jacob et F. Recanati: La logique des noms propres, Paris, Minuit, 1982).

LEVINSON, J. (1980), "The particularisation of attributes», Australasian Journal of Philosopby, 58, pp. 102-115.

LOUX, M.J. (1983), « Recent work in ontology», in Recent Work in Philosophy, sous la dir. de K.G. Lucey et T.R. Machan, Totowa, N.J., Rowman and Allanheld, p. 3-38.

MACE, C.A. (1967), "Stout, George Frederick», in The Encyclopedia of Philosophy, sous la dir. de P. Edwards, New York, Macmillan, vol. 8, pp. 22-24.

MATTHEWS, G.B. et COHEN, S.M. (1967-68), «The One and the Many », The Review of Metaphysics, 21, pp. 630-655. 
MOORE, G.E. (1962), «Are the characteristics of particular things universal or particular?", in Philosophical Papers (recueil d'articles de G.E. Moore), New York, Macmillan, pp. 17-31 ( $1^{\text {re }}$ éd. du recueil : 1959; public. orig. de l'article: Proceedings of the Aristotelian Society, supp. vol. 3, 1923).

OCCAM, Guillaume d' (1957), Philosophical writings, édition d'extraits en latin avec tr. angl. par P. Boehner, Edinburgh, Nelson.

(1967-86), Opera Philosophica et Theologica, 16 vols. répartis en deux séries: Opera Philosophica (6 vols.) et Opera Theologica (10 vols.), St. Bonaventure, N.Y., The Franciscan Institute.

(1969), Predestination, God's Foreknowledge, and Future Contingents, trad. angl. avec introd., notes et appendices par M.M. Adams et N. Kretzmann, New York, Appleton-Century-Crofts ( $2^{e}$ éd. avec nouvelle introd. par M.M. Adams : Indianapolis, Hackett, 1983).

(1973-74), "William of Ockham's Commentary on Porphyry», tr. angl. par E.H.W. Kliige, Franciscan Studies, 33, pp. 71-254, et 34, pp. 306-382.

(1974), Ockham's Theory of Terms. Part I of the Summa Logicae, introd. et tr. angl. par M.J. Loux, Notre-Dame, University of Notre Dame Press.

(1980), Ockham's Theory of Propositions. Part II of the Summa Logicae, introd. par A.J. Freddoso et tr. angl. par A.J. Freddoso et H. Schuurman, Notre Dame, University of Notre Dame Press.

OWEN, G.E.L. (1965), «Inherence », Phronesis, 10, pp. 97-105.

PANACCIO, C. (1972), « Guillaume d'Occam : logique et lecture», Critère, 6-7, pp. 95-110.

(1973), «La métaphysique et les noms», in Culture et langage, sous la dir. de J.P. Brodeur et G. Leroux, Montréal, H.M.H., pp. 249-281.

(1974), «Langage ordinaire et langage abstrait chez Guillaume d'Occam», Philosophiques, 1, pp. 37-60.

(1980), «Occam et les démonstratifs», Historiographia Linguistica, 7, pp. 189-200.

(1981), «Le commentaire de Guillaume d'Occam sur le livre des prédicables de Porphyre, introduction par Louis Valcke, traduction française par Roland Galibois », étude critique, Dialogue, 20, pp. 318-334.

(1983), "Guillaume d'Occam: signification et supposition», in Archéologie du signe, sous la dir. de Y. Brind'Amour et E. Vance, Toronto, Institut Pontifical d'Études Médiévales, pp. 265-286.

(1984), «Pour une théorie occamiste des conditions de vérité», in Matériaux pour une histoire des théories linguistiques, sous la dir. de S. Auroux et als., Lille, Université de Lille III, pp. 211-216.

(1985), «Der Nominalismus Ockhams und der zeitgenössische Nominalismus », trad. du franç. par L. Kaczmarek, in Rekonstruktion und 
Interpretation, sous la dir. de K.D. Dutz et L. Kaczmarek, Tübingen, Narr, pp. 1-22.

RUSSELL, B. (1956), Logic and Knowledge, éd. par R.C. Marsh, Londres, George Allen and Unwin.

(1961), Histoire de mes idées philosophiques, tr. franç. par G. Auclair, Paris, Gallimard (My Philosophical Development, Londres, George Allen and Unwin, 1959).

(1968), Autobiograpbie 1872-1914. Tr. franç. par M. Berveiller, Paris, Stock. (Autobiography, vol. 1, Londres, George Allen and Unwin, 1967).

STOUT, G.F. (1923), «Are the characteristics of particular things universal or particular? ", Proceedings of the Aristotelian Society, supp. vol. 3, pp. 114122.

(1936), «Universals again», Proceedings of the Aristotelian Society, supp. vol. 15, pp. 1-15.

(1966), "The nature of universals and propositions», in Studies in Philosophy, sous la dir. de J.N. Findlay, Oxford, Oxford University Press (public. orig. de l'article: Proceedings of the British Academy, 10, 1921).

WILLIAMS, D.C. (1953), "On the elements of Being", The Review of Metaphysics, 7, pp. 3-18 et 171-192 (repris in Principles of Empirical Realism, Springfield, Ill., Charles C. Thomas, 1966).

WOLTERSTORFF, N. (1970), On Universals, Chicago, Chicago University Press. 